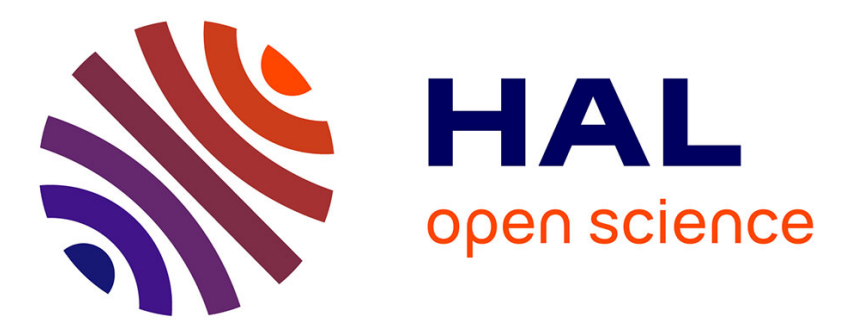

\title{
Hybrid polymer/lipid vesicles: fine control of the lipid and polymer distribution in the binary membrane
}

Maud Chemin, Pierre-Marie Brun, Sébastien Lecommandoux, Olivier Sandre, Jean-François Le Meins

\section{- To cite this version:}

Maud Chemin, Pierre-Marie Brun, Sébastien Lecommandoux, Olivier Sandre, Jean-François Le Meins. Hybrid polymer/lipid vesicles: fine control of the lipid and polymer distribution in the binary membrane. Soft Matter, 2012, 8 (10), pp.2867-2874. 10.1039/c2sm07188f . hal-00744120

HAL Id: hal-00744120

https://hal.science/hal-00744120

Submitted on 28 Mar 2019

HAL is a multi-disciplinary open access archive for the deposit and dissemination of scientific research documents, whether they are published or not. The documents may come from teaching and research institutions in France or abroad, or from public or private research centers.
L'archive ouverte pluridisciplinaire HAL, est destinée au dépôt et à la diffusion de documents scientifiques de niveau recherche, publiés ou non, émanant des établissements d'enseignement et de recherche français ou étrangers, des laboratoires publics ou privés. 


\title{
Hybrid polymer/lipid vesicles: fine control of the lipid and polymer distribution in the binary membrane
}

\author{
Maud Chemin, ${ }^{a, b}$ Pierre-Marie Brun, ${ }^{a, b}$ Sébastien Lecommandoux, ${ }^{a, b}$ Olivier Sandre ${ }^{* a, b}$ and \\ Jean-François Le Meins*a,b
}

Received 15th November 2011, Accepted 19th December 2011

DOI: $10.1039 / \mathbf{c} 2 \mathrm{sm07188f}$

Hybrid polymer/lipid giant unilamellar vesicles (GUV) were developed using lipids of respectively low and high melting transition temperature (DPPC:1,2-dipalmitoyl-sn-glycero-3 phosphocholine, $T_{\mathrm{m}}=41^{\circ} \mathrm{C}$, and POPC: palmitoyl-2-oleoyl-sn-glycero-3phosphocholine; $T_{\mathrm{m}}=-2^{\circ} \mathrm{C}$ ) and a copolymer poly(dimethylsiloxane)-graft-poly(ethylene oxide) (PDMS- $g$-PEO) well known to selfassemble into vesicular structures. Using epifluorescence microscopy as well as differential scanning calorimetry (DSC), different structures have been identified depending on the molar composition and on the fluid or gel state of the lipid used. The most promising objects are hybrid vesicles with copolymer as major component, in which lipids are either randomly distributed or present "raft-like" domains in the polymer-rich membrane. The results are discussed on the basis of the fluidity of the different components and of their respective membrane thickness.

\section{Introduction}

Liposomes developed in the early sixties ${ }^{1}$ constitute the first examples of synthetic analogues to biological cells, for which the membranes are also mainly constituted of lipids (glycerophospholipids, sphyngolipids, cholesterol, etc.). Biodegradable and non-toxic, liposomes are easily internalized by living cells and are able to encapsulate both hydrophobic and hydrophilic materials. Depending on the process used, multilamellar (MLV) or unilamellar (SUV, LUV and GUV) vesicles of different sizes $(50 \mathrm{~nm} \text { to } 100 \mu \mathrm{m})^{2}{ }^{3,4}$ can be obtained and used as drug carriers for intravenous administration for the smallest ones $(<200 \mathrm{~nm})$, or model objects for understanding cell membrane properties for the giant ones $(>10 \mu \mathrm{m})$. Pioneering work on mechanical and viscoelastic properties of liposome membranes has been realized by E. Evans ${ }^{5}$

The major drawback of liposomes is their low mechanical stability and leakiness, mainly due to their small membrane thickness $(3 \sim 5 \mathrm{~nm})$. They also present a limited chemical versatility leading to restrained applications. Several approaches to modify their membrane properties have been developed. For instance their resistance to opsonisation and blood clearance (stealthiness) has been improved by anchoring or grafting hydrophilic macromolecules -mainly poly(ethylene oxide)- at the surface of liposomes, but this can lower the cohesion of the lipid in bilayers and affect their stability. ${ }^{6}$ Increase of the membrane stiffness and decrease of lateral mobility can be achieved to some extent for instance by incorporating cholesterol. ${ }^{6}$

As another interest, lipid vesicles constitute an excellent tool for the understanding of biological events such as cell adhesion, signal transduction or endocytosis, in which the existence of heterogeneities or domains in biological membrane play an important role. ${ }^{7}$ Formation of domains in a membrane can result in a phase segregation process in the bilayer. For instance in a binary mixture of lipids, lateral phase separation can occur if the respective chain lengths of phospholipids differ by more than two carbons or if the lipid head groups are different (as with phosphatidylcholine and phosphatidylethanolamine). The formation of domains can also be triggered by external stimuli such as temperature, lateral pressure, adhesion or integration of macromolecules in the bilayer. ${ }^{6,8}$ A possibility of demixing was also described when mobile stickers anchored to lipids are recruited towards an adhesion $z^{2} \mathrm{e}^{9}$, a phenomenon also found with electrostatic adhesion of giant liposomes on solid substrate leading to membrane "blistering". ${ }^{10}$ Polymeric additives can also be used to modulate the membrane properties of the liposomes. For instance it has been established that pores of controlled size can be obtained by addition of amphiphilic cationic macromolecules called "amphipols", 6, 8, 11

Polymersomes present a similar structure to liposomes, but result from the self-assembly of amphiphilic block copolymers into monolayers (grafted and triblock copolymers) or bilayers (diblock copolymers). These structures are the object of increasing activities in the field of polymer science but also in the field of drug delivery since the last decade. The different ways to tune their membrane properties has been recently reviewed. ${ }^{12}$ Polymersomes open new opportunities for advanced functionalization and their membrane present outstanding stability. Most often, the polymersomes' membrane properties differ by more than one or two orders of magnitude from those of liposomes (shear viscosity, lateral diffusion coefficient, permeability...), having significant consequences on drug release properties, with kinetics shown to be at least 5 to 10 times slower than for liposomes ${ }^{13-17}$. However, one of the drawbacks of this increased thermodynamic stability is the difficulty for low molar mass species to diffuse into or out of the polymersomes' membrane. A way to circumvent this phenomenon consists in constructing stimuli responsive membranes, ${ }^{18,19-21}$ that either decompose or reversibly open and close upon exposure to a specific trigger, or by addition of channel proteins to the polymer membrane, ${ }^{22-25}$ this last approach being only possible for extremely flexible hydrophobic polymer blocks. 
Another recently developed approach to modulate vesicle membrane properties consists in blending phospholipids and block copolymers to engineer hybrid vesicles, which membrane is constituted of both components. The rationale consists in taking advantage of robustness and chemical versatility of polymer membranes, combined with the biocompatibility and softness of phospholipid membranes. This goal can be probably achieved by a cautious repartition of the lipids in the polymer membrane. Two limit cases are emerging: lipid molecules can be homogeneously distributed in the polymer membrane, or demixtion can occur leading to the formation of two phases with lipid-rich domains in the polymer-rich membrane. The way to control these two extreme situations has not been clearly evidenced in the literature as well as their consequences on their membrane physical properties and their biofunctionality. Pioneering work has been realized in the nineties by Kostarelos and col. ${ }^{26,27}$ who tried to elucidate the incorporation of triblock copolymers (Synperonics) in soybean lecithin vesicles by monitoring the absorbance change of different hydrophobic dyes solubilised in the liposomes bilayer upon the addition of small amount of copolymer. The formation of hybrid vesicle bilayers with this technique was hard to prove. Ten years later, Ruysschaert et al. ${ }^{28}$ showed that hybrid vesicles constituted of poly(2-methyloxazoline)-block-poly(dimethylsiloxane)-blockpoly(2-methyloxazoline) $\quad\left(\mathrm{PMOXA}_{1800}-b-\mathrm{PDMS}_{5400}-b\right.$ $\mathrm{PMOXA}_{1800}$ ) and egg-phosphatidylethanolamine or dipalmitoylphosphatidylcholine (DPPC) could be obtained. According to fluorescence quenching experiments, a homogeneous distribution of the lipid in the polymer membrane was proposed for low lipid content ( $\leq 20 \mathrm{~mol} \%$ ). DSC experiments for higher lipid content (up to $90 \mathrm{~mol} \%$ ) led the authors to the same conclusion. Cheng and Tsourkas ${ }^{29}$ prepared hybrid lipid polymer vesicles, but only as a necessary step (sacrificial template) to obtain porous polymersomes. No information about the hybrid membrane structure could be collected. In a very recent study, ${ }^{30}$ the same authors have formulated nanovesicles from the aqueous co-assembly of poly(ethylene oxide)-block-polybutadiene (PEO-b-PBD and phospholipid, and a moderated amount of hydrogenated soy phosphatidylcholine (HSPC) $(\leq 25 \mathrm{~mol} \%)$. They noted an improvement of the targeting of cancer cell, although no information was given about the either homogeneous or heterogeneous distribution of lipid in the membrane. The creation of "spot-like" lipid rich domain in hybrid lipid/polymer vesicle have been achieved by introducing an external driving force to perturb the initial equilibrium homogeneous state of hybrid vesicles composed of PEO- $b$-PBD and POPC. This was realized by cross-linking a fraction of biotinylated lipid head groups with the multifunctional protein Neutravidin. ${ }^{31}$ It has to be noted in this study that at low polymer content $(\leq 30 \mathrm{~mol} \%)$, the formation of hybrid vesicle was not energetically favorable because high molecular weight diblock copolymer molecules cannot be easily incorporated into the thinner lipid rich membrane. Moreover, there is a window of composition where no vesicles could be obtained (35-65 mol\% of polymer). In another recent study, hybrid lipid polymer vesicles with segregated membranes (domains) has been also obtained using a mixture of DPPC and poly(isobutylene)-block-poly(ethylene oxide) block copolymers with two different molecular weight. This was observed only in a narrow molecular composition window (20-28 mol\% of block copolymer) and only for block copolymers with a large hydrophobic block $(\sim 5000 \mathrm{~g} / \mathrm{mol})$, the shorter one giving hybrid homogeneous vesicles. Regarding such molar composition, liposomes containing "copolymer domains" were obtained. Globally, the conditions under which such hybrid structures are obtained are not well established: in particular the role of the molecular composition of the block copolymer and lipids and their respective molar proportion is not clearly understood and rationalized. For instance it is mentioned in the work of Nam et $\mathrm{al}^{31}$ that in a given molar composition range no vesicles were obtained, which is not mentioned in the work of Ruysschaert et $\mathrm{al}^{28}$ where a systematic study of the molar composition was conducted. Another important parameter is the respective length of the hydrophobic part of lipid and block copolymer. In all the studies performed, the block copolymer used lead to the formation of polymersomes with membrane thickness of at least $7 \mathrm{~nm},{ }^{12}$ which is above the average thickness of liposome membrane $(3 \sim 4 \mathrm{~nm})$. Ruysschaert et al. ${ }^{28}$ suggested that a large difference in hydrophobic lengths of lipid and polymer should favour homogeneous distribution of lipid (at a moderate molar \%) to prevent hydrophobic mismatch that would result from the formation of lipid domains of much smaller bilayer thickness.

It has to be noted that polymersomes resulting from the selfassembly of a blend of two copolymers, differing only by their hydrophilic block ${ }^{32}$ or by both hydrophobic and hydrophilic blocks and molar mass, ${ }^{33}$ can present phase separation of domains with distinct composition in the membrane. Segregated core or corona can be also obtained in so called "Patchy micelles" composed of different block copolymers. The different ways to develop such structures as well as their use in drug delivery have been recently reviewed. ${ }^{34}$

In this work, we propose to rationalize the effect of respective membrane thickness of the polymersomes and liposomes as well as the fluidity of the lipid used (either in gel state or in fluid state at temperature of interest) on the formation of hybrid vesicles and their resulting membrane structure. For that purpose, we will formulate mixed vesicles composed of a poly(dimethylsiloxane)graft-poly(ethylene oxide) copolymer PDMS-g-PEO, well known to form vesicular structure with a membrane thickness of $\sim 5 \mathrm{~nm}$ very close to that of liposomes, and two different phospholipids, DPPC and POPC, respectively in gel state and fluid state at room temperature.

\section{Materials and Methods}

Materials. PDMS- $g$-PEO is a gift from Dow Corning. It is composed of a PDMS chain decorated with two arms of PEO, in average, with a degree of polymerization of 12 . The weight fraction of ethylene oxide is $47 \%$ according to supplier data, and confirmed by ${ }^{1} \mathrm{H}$ NMR analysis. The average viscosimetric molecular weight is equal to $3000 \mathrm{~g} / \mathrm{mol}$ according to literature and supplier data 35,36 and has been verified by viscosity measurements. Previous studies reported a membrane core thickness of $5 \mathrm{~nm}$, as measured by Cryo-TEM. ${ }^{37}$ We confirmed this value with the same technique. All these characterisations are reported in the Supporting Information of a previously published paper. $^{38}$

This copolymer was labelled with fluorescein (PDMS-g-PEOfluo) following a procedure described in Supporting Information. DPPC (1,2-dipalmitoyl-sn-glycero-3-phosphocholine) and POPC (1-palmitoyl-2-oleoyl-sn-glycero-3-phosphocholine) as well as L$\alpha$-phosphatidylethanolamine-N-(lissamine rhodamine B sulfonyl) (Liss-Rhod PE) were purchased from Avanti Polar lipids and used without further purification.

Formation of Vesicles. Giant unilamellar vesicles (GUV) were obtained using the electroformation procedure. ${ }^{3}$ Briefly, $1 \mathrm{mg}$ of the polymer/lipid mixture containing $200 \mathrm{nM}$ of Liss-Rhod PE 
and $6.4 \mu \mathrm{M}$ of PDMS- $g$-PEO-fluo was dissolved in $1 \mathrm{~mL}$ of chloroform/methanol (2/1), a solvent mixture chosen for its higher polarity and lower volatility than pure chloroform. Around $75 \mu \mathrm{l}$ of this solution was spread slowly with a syringe on both windows of a homemade preparation chamber composed of two transparent electrodes (glass plates coated with indium tin oxide, resistivity $15-25 \Omega /$ square) and a rubber spacer ( $1.5 \mathrm{~mm}$ thick). After drying under vacuum during $30 \mathrm{~min}$, the chamber was connected to AC voltage and filled with a solution of sucrose at $0.1 \mathrm{M}$ to tune osmolarity. A sinusoidal tension $(2 \mathrm{~V}, 10 \mathrm{~Hz})$ was applied for $20 \mathrm{~min}$ followed by $10 \mathrm{~min}$ under squared tension $(1 \mathrm{~V}, 2 \mathrm{~Hz})$. Solution was then extracted using syringe with $\geq 0.8$ $\mathrm{mm}$ internal diameter needle to minimize shear stress. Then a few drops were deposited in an Eppendorf ${ }^{\mathrm{TM}}$ tube containing $2 \mathrm{~mL}$ of glucose solution $(0.1 \mathrm{M})$ in order to collect a fraction at the bottom of this tube enriched with the largest sizes vesicles filled with sucrose suspended in glucose, after a few minutes to allow their sedimentation.

Vesicles analysis by optical microscopy. A Zeiss Axiovert 40 inverted microscope equipped with a digital Gigabit Ethernet camera (VG-2M Vieworks Ltd) for rapid recording of images (up to $100 \mathrm{fps}$ ) was used. The vesicles were observed either with a $\times 40 / 0.7$ or $\times 100 / 1.3$ (magnification/numerical aperture) objective lens working in epifluorescence mode. Excitation is provided by a mercury lamp. Different filter set were used in order to detect signal from fluorescein (FITC-3540B from Semrock, Em 513$560 \mathrm{~nm}$ ) or Rhodamine lissamine (Em 573/637), or both: triple band filter set (Em 448-478; Em 515-552; Em 600-663)

Differential scanning calorimetry. DSC analyses were performed on vesicle suspensions in sucrose $0.1 \mathrm{M}$ (concentration range from 4 to $15 \mathrm{wt} \%$ ) obtained by classical film rehydration, with a TA Instruments Q100 apparatus at a rate of $5^{\circ} \mathrm{C} / \mathrm{min}$ in sealed aluminium capsules. More details are given in Supporting Information.

\section{Results and discussion}

\section{Hybrid vesicles with PDMS-g-PEO and POPC.}

Vesicles prepared for molar compositions ranging from $0 \%$ to $100 \%$ of copolymer (with $10 \mathrm{~mol} \%$ increments) were studied both by DSC and microscopy. Fraction of $75 \%$ and $25 \%$ were also used for microscopy observations only. As the mixtures contained some amount of PDMS- $g$-PEO-fluo and Liss Rhod PE, we were able to distinguish directly the signal arising from copolymer and lipid phases in the membrane. The vesicles' suspension were observed first a few minutes after the electroformation process and then re-observed several hours or days after. When lipids were the major component of the mixture, vesicles were obtained and could be easily observed (Figure 1). The figure is divided in three parts $\mathrm{A}, \mathrm{B}, \mathrm{C}$ which correspond to observations made with different filter sets of the same sample area: Fluorescein filter (A), triple band filter (B) and rhodamin filter (C). Part of the vesicles (see for instance vesicle $N^{\circ} 1$ ) were visible only with $\mathrm{A}$ and $\mathrm{B}$ but almost invisible with $\mathrm{C}$. This reflects the fact that such vesicles were essentially polymersomes (containing possibly a very small amount of lipid). On the opposite, other vesicles $\left(\mathrm{N}^{\circ} 3\right)$ were visible in red with the triple band (B) and in yellow with the Rhodamin filters (C) but were not visible with the Fluorescein filter (A): they can be considered as (almost) pure liposomes. Interestingly, some vesicles $\left(\mathrm{N}^{\circ} 2\right)$ present unambiguously both signals (red and green) on different parts of their membrane. The red area (vesicle $\mathrm{N}^{\circ} 2$ ) on Figure 1$\mathrm{B}$ appears totally dark on figure 1-A. On figure 1-C, this is the opposite, the previously red area on vesicle $\mathrm{N}^{\circ} 2$ appears bright and the signal is almost extinguished (although a very weak signal of rhodamin can be detected) on the area corresponding previously to green. Therefore these vesicles presented clearly an inhomogeneous distribution of lipids and copolymer within the membrane.

The analysis of the same sample at different times allowed the observation of membrane budding: after a while, the domains form two spherical caps presenting different curvatures, connected to each other along a circular neck (Figure 2-B,C). Over time, the neck vanished (Figure 2-D) and complete fission occurred between two daughter vesicles adhering to each other on a large flat portion of membrane. At the end, a polymersome and a liposome were totally separated, as illustrated on Figure 2-E. The total kinetics of this phenomenon was rather slow, within hours. As a consequence, monitoring this fission process on the same vesicle was too delicate to achieve because of convection and bleaching phenomena.

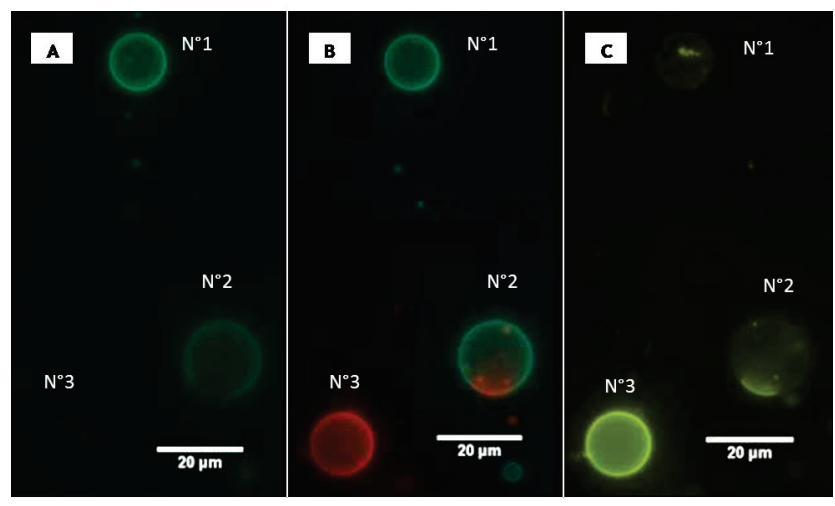

Fig. 1: Fluorescence microscopy pictures of vesicles obtained after electroformation process (75\% POPC $/ 25 \%$ PDMS- $g$-PEO). A:

Fluorescein filter set; B: Triple band filter set; C: Rhodamin filter set.
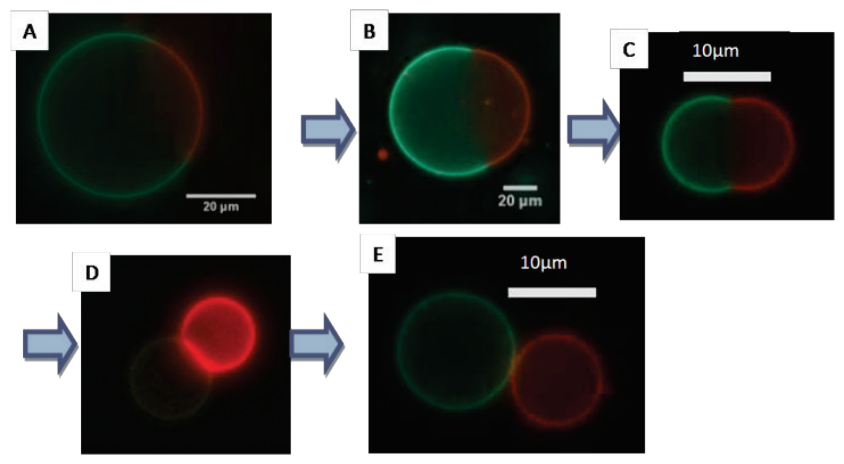

Fig. 2: Typical evolution over time (several hours) of a hybrid vesicle (75\% POPC/25\% PDMS- $g$-PEO), presenting domains . (Triple band filter set). A: initially spherical biphasic vesicle; B, C: the two domains present different curvatures within the same vesicle; $D$ : apparition of a

flat contact area between two adhering but separated vesicles; E: a polymersome (green) and a liposome (red) are now totally distinct

However, the evolution illustrated on Figure 2 obtained with different vesicles pictured at different times is representative of 
the same event that occurred for many vesicles in the sample. At around $50 \mathrm{~mol} \%$ of polymer and POPC, vesicles with domains were observed almost exclusively a few minutes after electroformation, leading to pure polymersomes and liposomes a few hours later. When polymer was the major component (60 to $90 \mathrm{~mol} \%$ ), the obtained vesicles were homogeneous and no domains could be clearly observed. Both fluorescent signals of Rhodamin labelled lipids and Fluorescein labelled polymers were detected as illustrated on Figure 3 whatever the filter set selected. These vesicles were stable over time (at least one week), neither phase separation into domains nor budding were observed.

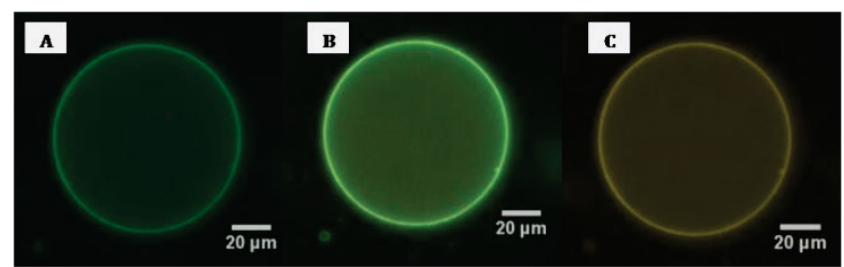

Fig. 3: Hybrid homogeneous vesicles obtained for the composition .25\% POPC $/ 75 \%$ PDMS- $g$-PEO. A: Fluorescein filter set. B: Triple band filter set. C: Rhodamine filter set.

To get more insight into the membrane phase behaviour, we performed differential scanning calorimetry (DSC) experiments on vesicular suspensions of POPC and copolymer. The thermograms can be consulted in ESI Fig. S2.

The main chain phase transition of POPC $\left(\mathrm{L}_{\beta}\right.$ to $\left.\mathrm{L}_{\alpha}\right)$ is observed at around $-4^{\circ} \mathrm{C}$. Due to peak broadness, the melting temperature was defined not as the peak itself but rather as the onset temperature (cross-over between tangent line at the inflection point with baseline) to evaluate the influence of the molar composition on $\mathrm{T}_{\text {onset. }}$ The surface area of the peak equal to melting enthalpy was also studied. The results are summarized on Figures 4 and 5.

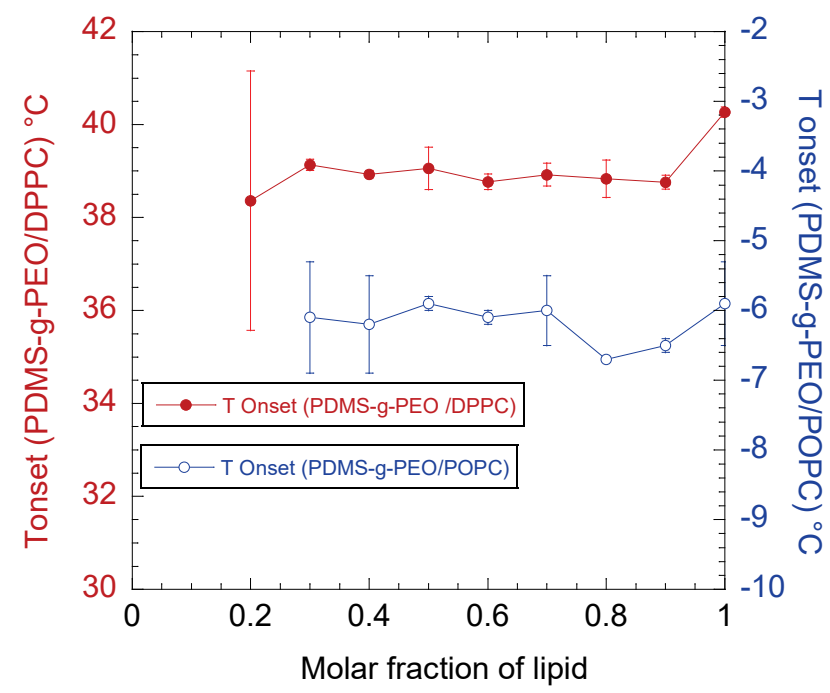

Fig. 4: Evolution of the main chain transition temperature of the lipid phase for vesicular suspension of PDMS- $g$-PEO/DPPC or POPC mixtures.

Globally, the onset melting temperature (Figure 4) remained unchanged (within error bars) compared to pure POPC whatever the molar composition although the values obtained at 0.8 and 0.9 seem to be slightly lower. When lipid was the major component of the mixture (50 mol\% and above), the suspensions must contain essentially separated liposomes and polymersomes as supported from microscopy observations (the DSC analysis being performed after hydration overnight which is sufficient to induce budding). Therefore the melting enthalpy and onset temperature corresponded to those of pure liposomes as observed experimentally. At molar lipid fractions $40 \mathrm{~mol} \%$ and $30 \mathrm{~mol} \%$ for which homogeneous hybrid vesicles were observed by fluorescence microscopy, the onset temperature was also unchanged. In a previous study with another copolymer $\left(\mathrm{PMOXA}_{1800}-b-\mathrm{PDMS}_{5400}-b-\mathrm{PMOXA}_{1800}\right)$ and lipids, such an invariance of the melting temperature was also observed. ${ }^{28} \mathrm{~A}$ melting transition could be detected up to $70 \mathrm{~mol} \%$ of copolymer in the mixture. Considering the uncertainty of the measurements, the melting enthalpy remained almost unchanged at high lipid fraction (100-70 mol\%) around $30 \mathrm{~J} / \mathrm{g}$. At $60 \mathrm{~mol} \%$ and $50 \mathrm{~mol} \%$ of lipid, the enthalpy decreases to $\sim 25 \mathrm{~J} / \mathrm{g}$ and continues to decrease upon further addition of copolymer down to $\sim 15 \mathrm{~J} / \mathrm{g}$, i.e. half the value of the pure lipid.

In all cases, the melting temperature did not change, thereby proving that the lipid in the gel phase was pure (not contaminated by polymer). Therefore we interpret the decrease of the melting enthalpy at low lipid content linked to a progressive "dilution" of the lipids in the polymer membrane (as expected from the images at $25 \mathrm{~mol} \%$ POPC showing homogeneous fluorescence). Such an effect may result in a decrease of cooperativity of the phase transition and thus of the melting enthalpy. Above $70 \mathrm{~mol} \%$ in polymer, the melting transition of lipid totally disappears, the lipid chains being unable to meet and nucleate the gel phase

It has to be noted that contrary to a previous study performed on mixtures of POPC and PBD- $b$-PEO, ${ }^{31}$ vesicles could be obtained whatever the molar composition in these PDMS-g-PEO/POPC formulation.

\section{Hybrid vesicles with PDMS-g-PEO and DPPC.}

In order to have a better understanding of the role of the fluidity of the lipid component in the formation of hybrid vesicles, we have followed exactly the same methodology using DPPC, which is in the gel state at room temperature.

As observed for PDMS- $g$-PEO/POPC, three different types of vesicles were also obtained when DPPC was the main component: polymersomes, liposomes and hybrid vesicles which presented clearly lipid and copolymer domains (Figure 6). The proportion of pure liposomes varied accordingly to the lipid content. The proportion of hybrid vesicles increased as composition approached isomolarity. It is worth noticing that we did not observe holes in the membrane of DPPC vesicles as mentioned in a very recent study using PIB- $b$-PEO and DPPC mixtures. $^{39}$ 


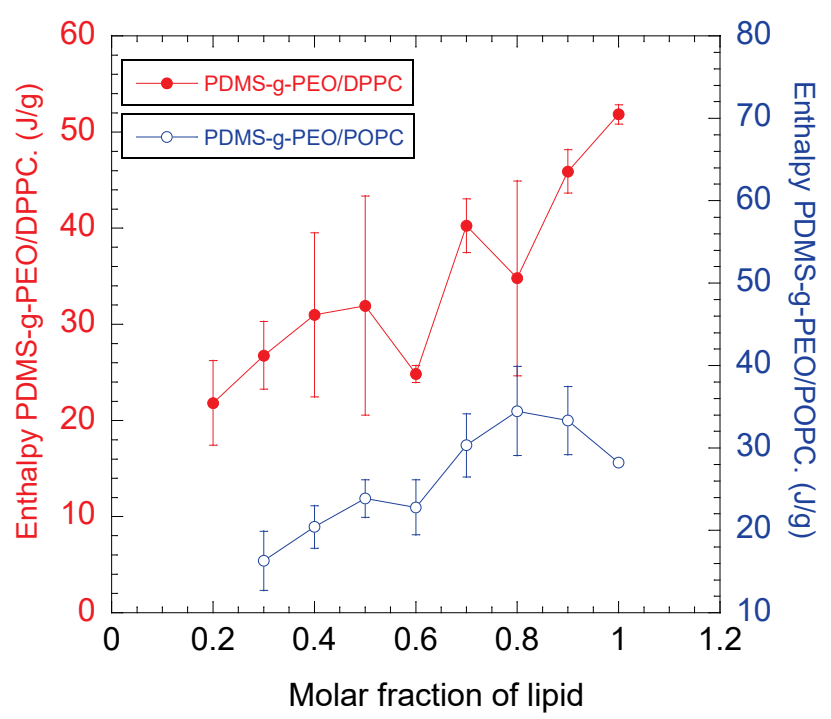

Fig. 5: Evolution of the melting enthalpy of the lipid phase for vesicular suspensions of PDMS- $g$-PEO/DPPC or POPC mixtures.

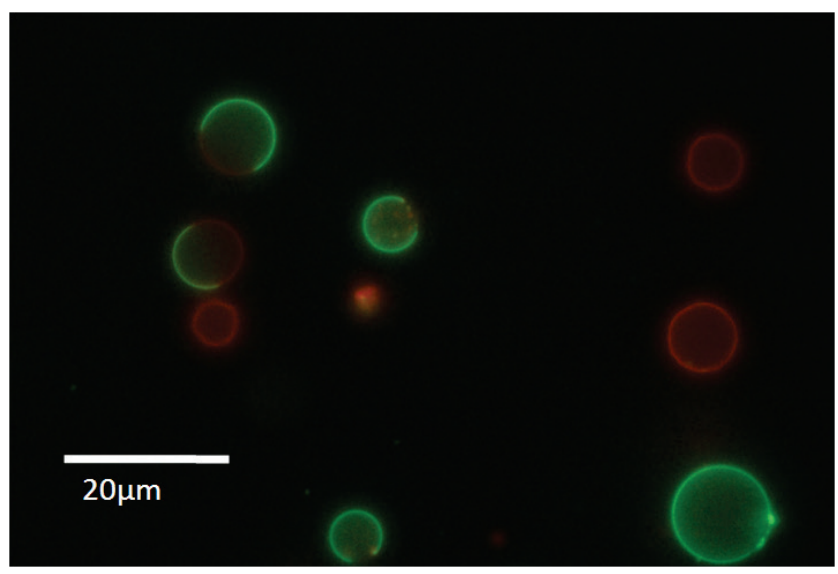

Fig 6: Fluorescence microscopy pictures of hybrid vesicles obtained after electroformation process ( $75 \%$ DPPC/25\% PDMS-g-PEO) with the tripleband filter set.

At $50 \mathrm{~mol} \%$ of copolymer and DPPC, only heterogeneous hybrid vesicles could be observed although it appeared that each vesicle did not contain systematically $50 \%$ of each component (Figure 7). In the case of polymersomes (green), a red component was visible in the green domain choosing the appropriate set filter, meaning that some lipid was dispersed in the mainly polymer membrane. The opposite was not clearly observed, although it could not to be excluded. (ESI, Fig. S4). When polymer was the major component, heterogeneous vesicles were still exclusively obtained, which constitute to the best of our knowledge the first experimental evidence of polymersomes containing a moderate or small proportion of lipids as domains obtained spontaneously, without external trigger (Figure 8-1). Unlike heterogeneous vesicles obtained with POPC, the hybrid vesicles made of PDMS$g$-PEO and DPPC were stable for several hours at room temperature. As with PDMS- $g$-PEO/POPC hybrid vesicles, we have also studied by DSC the melting transition of the lipid phases in hybrid vesicles suspensions composed of PDMS- $g$-PEO and DPPC. Thermograms can be consulted in ESI Fig. S1.

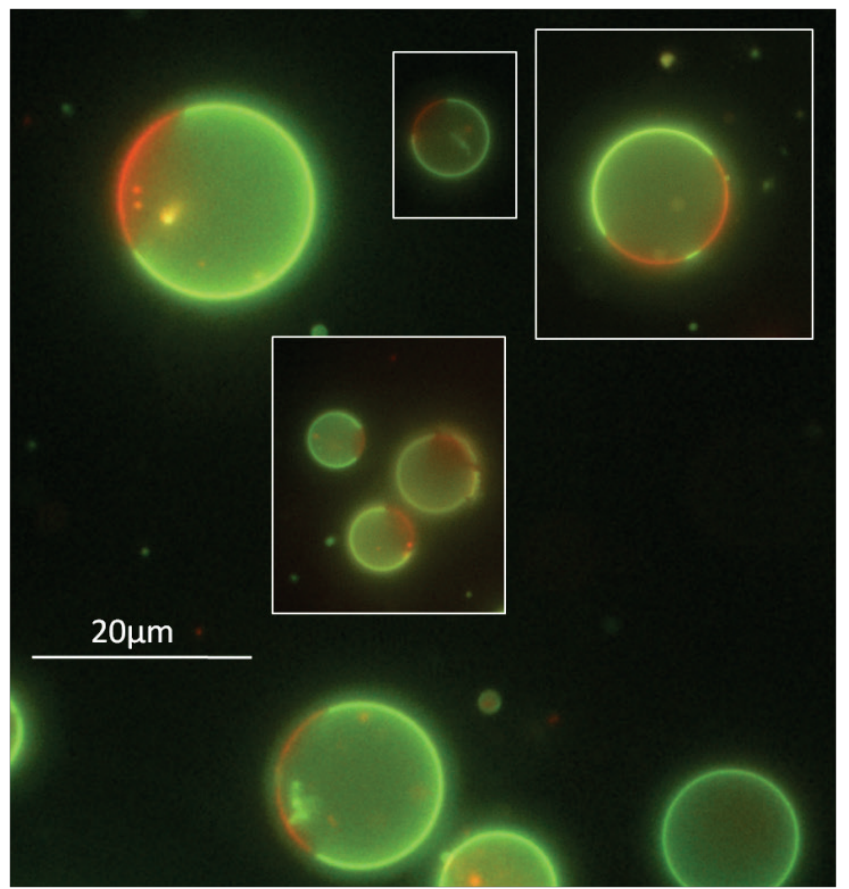

Fig.7 Hybrid heterogeneous vesicles obtained with 50\% DPPC and 50\% PDMS- $g$-PEO remaining stable for several days.

Once polymer was added, the shape of the melting transition was modified (peak broadening). Whereas the onset temperature decreased significantly (by $1{ }^{\circ} \mathrm{C}$ ) as soon as $10 \mathrm{~mol} \%$ was added relatively to pure DPPC, it did not decrease further when the copolymer content was increased. However, the enthalpy associated to the melting transition decreased upon copolymer addition in an almost linear manner (Figure 5). Below $20 \mathrm{~mol} \%$ lipid no melting could be detected.

This minimum amount of $20 \mathrm{~mol} \%$ lipid to detect a gel phase melting transition by DSC can be ascribed to the lipid solubility in the polymer-rich phase. It can be seen on Figure 8-2 that below this threshold, all lipid molecules are totally soluble in the polymer membrane. Therefore they cannot segregate into a lipidrich phase experiencing the $L_{\alpha}-$ to $-L_{\beta}$ phase transition. At $25 \mathrm{~mol}$ $\%$ of DPPC, red fluorescent domains could be detected, signifying that the lipid can segregate into lipid-rich domain. In other samples, domains excluding the green fluorescence of the labeled copolymer could also be detected, but without presenting themselves the red fluorescence of the rhodamin-labeled lipid (ESI Fig. S5).

These observations by fluorescence microscopy are correlated with the DSC results showing the presence of the fusion peak above $20 \mathrm{~mol} \%$ lipid. The copolymer interaction with the lipid manifests itself by its impact on the enthalpy as well as on the melting temperature for fractions as low as $10 \mathrm{~mol} \%$ polymer, thereby evidencing the dispersion of a part of copolymer chains within the lipid phase. Upon further addition of copolymer, the temperature of the melting transition remains unchanged, illustrating a "saturation" of the polymer in the lipid phase. However the enthalpy continues to decrease as the copolymer fraction increases, reaching a value of $\sim 20 \mathrm{~J} / \mathrm{g}$ at $20 \mathrm{~mol} \% \mathrm{lipid}$. 

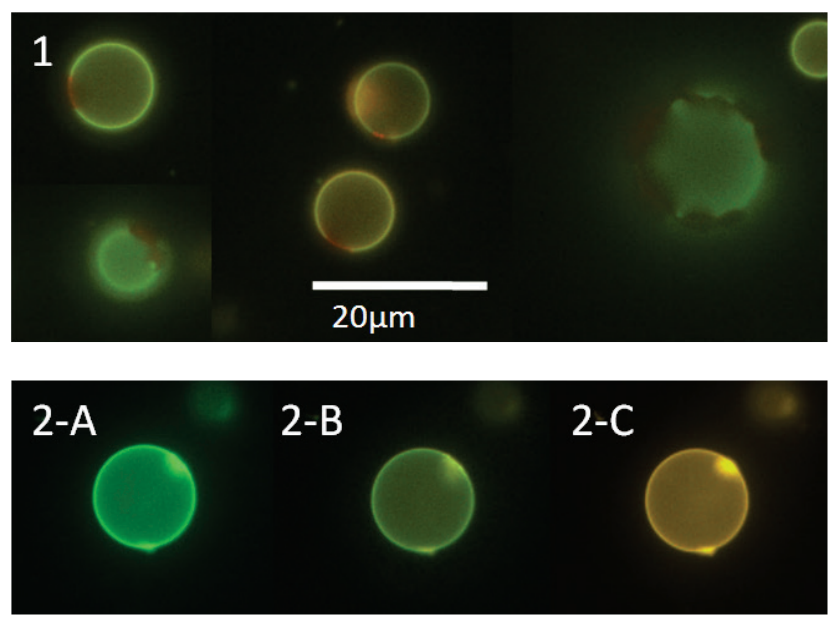

Fig. 8: Fluorescence microscopy pictures of lipid-polymer hybrid vesicles obtained by electroformation with the gel phase lipid DPPC at two compositions respectively above and below the $20 \mathrm{~mol} \%$ thereshold; 1 : heterogeneous case at 25\% DPPC/75\% PDMS- $g$-PEO observed with triple band filter set (In this case the concentration of Liss-Rhod PE was fixed at $120 \mathrm{nM}) ; 2$ : homogeneous case at $15 \%$ DPPC/85\% PDMS- $g$ PEO) (A: Fluorescein filter. B: Triple band filter. C: Rhodamin filter).

Although lipid domains are still present as revealed by their melting transition, less and less lipid molecules can be recruited in the cooperative $L_{\alpha}-$ to $-L_{\beta}$ phase transition because of a progressive dilution of the lipid into the polymer phase (see for instance ESI Fig. S4). Therefore 0.2 is the lowest lipid fraction at which domains of lipids can be obtained in a polymersome membrane, and thus can be viewed as a critical solubility value of DPPC inside the polymer. Below $20 \mathrm{~mol} \%$, no melting transition is detected, illustrating the fact that the minimum cooperative unit necessary to nucleate the gel phase of DPPC is not reached. This is confirmed by fluorescence microscopy showing only hybrid homogeneous vesicles on all fluorescence channels at $15 \mathrm{~mol} \%$ lipid (Figure 8-2).

\section{Discussion.}

Considering all these results, it is rather clear that the respective hydrophobic lengths of the copolymer and of lipid, which constitute the membrane, play a role in the success to achieve hybrid vesicles. Whatever the low or high gel-liquid crystalline transition temperature of lipid indeed, hybrid vesicles could be obtained over the whole composition range. As mentioned before, these results are significantly different from those obtained in a previous study which aimed to develop hybrid vesicles from POPC and poly(butadiene)- $b$-poly(ethylene oxide). ${ }^{31}$ In that case the polymersome hydrophobic thickness was close to $10 \mathrm{~nm}$. Practically no hybrid vesicles were obtained at high lipid content and even no vesicles at all could be obtained between 35 and 65 mol\% of lipid. The first point was explained in term of not energetically favourable incorporation of high molecular weight block copolymer molecule into the thin lipid-rich membrane, while the second point was not commented. In the case of the thinner PDMS-g-PEO grafted copolymer membranes, hybrid vesicles presenting lipid-rich and copolymer-rich domains are obtained at all lipid contents higher than $50 \mathrm{~mol} \%$ for POPC and $20 \mathrm{~mol} \%$ for DPPC. In the former case, with a main chain transition below room temperature, these biphasic vesicles remain stable for a few hours, before giving birth to pure liposomes and pure polymersomes by budding and fission processes. The phenomenon is similar to what is observed for mixtures of lipids exhibiting the coexistence of two phases which both are fluid. ${ }^{40,41}$ It can be reasonably assumed that the bending elasticity moduli of PDMS- $g$-PEO and POPC membranes are different. Therefore the membrane curvature energy can be modulated by lateral phase separation. The fluid domains then undergo a budding process (Figure 2) driven by the balance between the mean curvature energy and the line tension. This energetic cost of the domain boundary most probably arises from the compositional heterogeneity at the phase boundary, rather than from a hydrophobic mismatch between coexisting domains in our case (the hydrophobic thicknesses of lipid and copolymer being nearly equal). During the transformation between an incomplete to a complete bud, the neck becomes narrower and the domain has to stretch the membrane. ${ }^{42}$ This is made possible by the large excess area present of the membrane as both PDMS- $g$-PEO and POPC are in a fluid state at ambient temperature. When the copolymer is the major component (60 to $90 \mathrm{~mol} \%$ ), stable homogeneous vesicles are obtained and no subsequent growth of lipid domains was observed. This suggests that a critical lipid domain size needs to be reached in the vesicle during its formation by the electroformation process, to grow further by migration of lipid and to reach a size observable by microscopy.

With a lipid in a gel state at room temperature such as DPPC, heterogeneous vesicles stable over time are obtained in a large range from 90 to $20 \mathrm{~mol} \%$ of lipid. Therefore polymersomes presenting few domains of lipid can be obtained. Here the gel state of the lipid prevents the budding phenomenon previously observed with POPC. It is interesting to note that domains of lipid can be obtained down to $20 \mathrm{~mol} \%$ of lipid, whereas homogeneous vesicles were obtained using POPC once its content decreases below $50 \mathrm{~mol} \%$. This illustrates the role played by the fluidity of lipid on the stability of the formed hybrid vesicles, but also on their membrane structuration. We believe that the neighbouring thickness of the respective copolymer and lipid membranes and the gel state of the lipid are determinant parameters to obtain heterogeneous hybrid vesicles and in particular polymersomes presenting lipid-rich domains.

\section{Conclusions and perspectives}

Scheme 1 summarizes the different structures obtained in this study depending on composition and lipid fluidity. In this work, hybrid giant unilamellar vesicles composed of an amphiphilic copolymer (PDMS-g-PEO) and zwitterionic phospholipids (POPC, DPPC) were developed using the electroformation process. We aimed at bringing new elements for the understanding of the structuration process of such hybrid membranes. A methodology to obtain systematically polymersomes presenting lipid-rich domains is particularly interesting in the perspective of the incorporation of membrane proteins into vesicles, which would combine the features of polymersomes and liposomes. In that context, we choose a copolymer with a fluid character, well-known to form vesicles with a membrane thickness close to that of liposomes, in order to prevent a hydrophobic mismatch at the domain boundary and thus facilitate their formation by the natural phase separation expected between PDMS chains and lipid tails. 


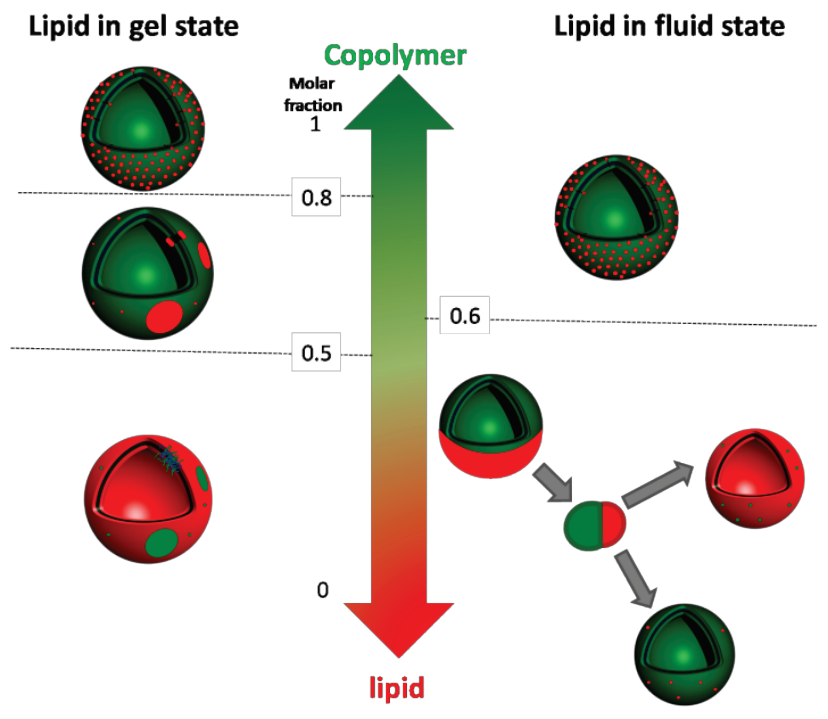

Scheme 1: An overview of the different obtained hybrid vesicles according to the molar composition and fluidity of the lipid at room temperature

Whereas hybrid vesicles with lipid-rich and copolymer-rich phases can be obtained when a fluid lipid is the major component, these vesicular structures undergo a budding process that finally leads to the formation of separated polymersomes and liposomes within a few hours. When the copolymer is mixed as the major component ( $\geq 60 \mathrm{~mol} \%$ ) with a fluid lipid (POPC), the obtained hybrid vesicles can be viewed as polymersomes in which lipids are homogeneously dispersed. The behaviour is completely different when the lipid used is in the gel state (DPPC). Heterogeneous hybrid vesicles are obtained for any composition between 90 and $20 \mathrm{~mol} \%$ of lipid. These are stable over time, the budding process being hampered by the gel character of the lipid domains. At copolymer content between $50 \mathrm{~mol} \%$ and $80 \mathrm{~mol} \%$, heterogeneous vesicles are obtained exclusively. This is to our knowledge the first experimental evidence that polymersomes with lipid-rich domains can be spontaneously obtained, without any external trigger. Below $20 \mathrm{~mol} \%$ of lipid, homogeneous hybrid vesicles are also observed.

All these structures might present an outstanding interest in the fields of drug delivery, micro- or nano-reactors, and more generally as biomimetic models. For instance mixtures of fluid lipid and copolymer can be useful as physical models to get more insight into the mechanisms of budding and fission, which are of prime importance to understand biological phenomena in cellular membrane trafficking. A total control of the molar composition in each vesicle, in order to precisely tune the properties of a whole population of vesicles still remains a challenge that will be addressed in the forthcoming years.

\section{Notes and references}

a Univ. Bordeaux, LCPO, UMR 5629, F-33600 Pessac, France.

b CNRS, LCPO, UMR 5629, F-33600 Pessac, France.
${ }^{\mathrm{b}}$ CNRS, Laboratoire de Chimie des Polymères Organiques (UMR5629), Pessac, France

*Correspondence to OS: E-mail: osandre@enscbp.fr; Tel: +33-5-56847947;JFLM: E-mail:lemeins@enscbp.fr; Tel: +33 556846194

$\dagger$ Electronic Supplementary Information (ESI) available: DSC curves representing the heat flow $(\mathrm{mW}) v s$. temperature $\left({ }^{\circ} \mathrm{C}\right)$ for the different systemes studied. Synthesis procedure and characterisation of PDMS-gPEO-fluo. Pictures illustrating the presence of lipids in the polymer phase. https://dx.doi.org/10.1039/c2sm07188f

1. A. D. Bangham, M. M. Standish and N. Miller, Nature, 1965, 208, 1295-1297.

2. S. Manley and V. D. Gordon, Current Protocols in Cell Biology, 2008.

3. M. I. Angelova and D. S. Dimitrov, Faraday Discussions of the Chemical Society, 1986, 81, 303-311.

4. D. J. Estes and M. Mayer, Biochimica et Biophysica Acta Biomembranes, 2005, 1712, 152-160.

5. E. Evans, K. Ritchie and R. Merkel, Biophysical Journal, $1995,68,2580-2587$.

6. C. Tribet and F. Vial, Soft Matter, 2007, 4, 68-81.

7. R. G. Anderson and K. Jacobson, Science, 2002, 296, 18211825.

8. W. H. Binder, V. Barragan and F. M. Menger, Angew. Chem. Int. Ed., 2003, 42, 5802-5827.

9. F. Brochard-Wyart and P. G. de Gennes, Proc. Natl. Acad. Sci., 2002, 99, 7854-7859.

10. J. Nardi, R. Bruinsma and E. Sackmann, Physical Review E, 1998, 58, 6340-6354.

11. W. H. Binder, Angew. Chem. Int. Ed., 2008, 47, 3092-3095.

12. J. F. Le Meins, O. Sandre and S. Lecommandoux, Eur. Phys. J. E, 2011, 34 (2), 14.

13. D. Peer and R. Margalit, Int. J. Cancer, 2004, 108, 780-789.

14. S. Suarez, R. J. Gonzalez-Rothi, H. Schreier and G. Hochhaus, Pharm. Res., 1998, 15, 461-465.

15. F. Ahmed, R. I. Pakunlu, A. Brannan, F. Bates, T. Minko and D. E. Discher, J. Control. Release, 2006, 116, 150-158.

16. F. Meng, G. H. M. Engbers and J. Feijen, J. Control. Release, 2005, 101, 187-198.

$17 . \quad$ Z. Pang, W. Lu, H. Gao, K. Hu, J. Chen, C. Zhang, X. Gao, X. Jiang and C. Zhu, J. Control. Release, 2008, 128, 120-127. M. H. Li and P. Keller, Soft Matter, 2009, 5, 927-937.

19. E. Mabrouk, S. Bonneau, L. Jia, D. Cuvelier, M.-H. Li and P. Nassoy, Soft Matter, 2010, 6, 4863-4875.

$20 . \quad$ N. P. Kamat, G. P. Robbins, J. Rawson, M. J. Therien, I. J. Dmochowski and D. A. Hammer, Adv. Funct. Mater., 2010 20, 2588-2596.

21. E. Mabrouk, D. Cuvelier, F. Brochard-Wyart, P. Nassoy and M. H. Li, Proc. Natl. Acad. Sci., 2009, 106 7294-7298

22. M. Kumar, M. Grzelakowski, J. Zilles, M. Clark and W. Meier, Proc. Natl. Acad. Sci., 2007, 104, 20719-20724.

23. A. Ranquin, W. Versées, W. Meier, J. Steyaert and P. Van Gelder, Nano Letters, 2005, 5, 2220-2224.

$24 . \quad$ M. Sauer, T. Haefele, A. Graff, C. Nardina and W. Meier, Chem. Commun., 2001, 2452-2453.

$25 . \quad$ C. Nardin, S. Thoeni, J. Widmer, W. Mathias and W. Meier, Chem. Commun., 2000, 1433-1434.

26. K. Kostarelos, P. F. Luckham and T. F. Tadros, J. Lipid Res., $1995,5,117-130$

27. K. Kostarelos, P. F. Luckham and T. F. Tadros, J. Lipid Res., $1995,5,443-452$

$28 . \quad$ T. Ruysschaert, A. F. P. Sonnen, T. Haefele, W. Meier, M. Winterhalter and D. Fournier, Journal of the American Chemical Society, 2005, 127, 6242-6247.

29 Z. Cheng and A. Tsourkas, Langmuir, 2008, 24, 8169-8173.

30. Z. Cheng, D. R. Elias, N. P. Kamat, E. Johnston, A. A. Poloukhtine, V. V. Popik, D. A. Hammer and A. Tsourkas, Bioconjugate Chem., 2011, doi.org/10.1021/bc200214g.

31. J. Nam, P. A. Beales and T. K. Vanderlick, Langmuir, 2011, 27, 1-6.

32. D. A. Christian, A. Tian, W. G. Ellenbroek, I. Levental, K. Rajagopal, P. A. Janmey, A. Liu, T. Baumgart and D. E. Discher, Nature Mater., 2009, 8, 843-849. 
33. C. Lopresti, M. Massignani, C. Fernyhough, A. Blanazs, A. J. Ryan, J. Madsen, N. J. Warren, S. P. Armes, A. L. Lewis, S. Chirasatitsin, A. J. Engler and G. Battaglia, ACS nano, 2011, $5,1775-1784$.

34. A. B. E. Attia, Z. Y. Ong, J. L. Hedrick, P. P. Lee, P. L. R. Ee, P. T. Hammond and Y.-Y. Yang, Current Opinion in Colloid \& Interface Science, 2011, 16, 182-194.

35. R. M. Hill, Langmuir, 1993, 9, 2789-2798.

36. J. Nam and M. M. Santore, Langmuir, 2007, 23, 7216-7224.

37. Z. Lin, R. M. Hill, H. T. Davis, L. E. Scriven and Y. Talmon, Langmuir, 1994, 10, 1008-1011.

38. A. Carlsen, N. Glaser, J.-F. Le Meins and S. Lecommandoux, Langmuir, 2011, 27, 4884-4890.

39. M. Schulz, D. Glatte, A. Meister, P. Scholtysek, A. Kerth, A. Blume, K. Bacia and W. H. Binder, Soft Matter, 2011, 7, 8100-8110.

40. R. Lipowsky, Curr. Opin. Struct. Biol., 1995, 5, 531-540.

41. S. Semrau and T. Schmidt, Soft Matter, 2009, 5, 3174-3186.

42. R. Lipowsky, Biophys. J., 1993, 64, 1133-1138. 


\title{
Hybrid polymer/lipid vesicles: fine control of the lipid and polymer distribution in the binary membrane
}

\author{
Maud Chemin, ${ }^{a, b}$ Pierre-Marie Brun, ${ }^{a, b}$ Sébastien Lecommandoux, ${ }^{a, b}$ Olivier Sandre ${ }^{\star a, b}$ and \\ Jean-François Le Meins* ${ }^{* a, b}$

\section{DSC Experiments}

A given appropriate volume of polymer/lipid solution in chloroform corresponding to a constant mass of lipid $(0.5 \mathrm{mg})$ was deposited in an aluminium pan, the mass of polymer being adapted to get the desired molar ratio. The solutions were evaporated under vacuum at $45^{\circ} \mathrm{C}$ during at least $5 \mathrm{~h}$ and then rehydrated with an aqueous solution of sucrose at $0.1 \mathrm{M}$ overnight. For lipid polymer mixture with DPPC, the first $30 \mathrm{mn}$ of hydration were performed at $50^{\circ} \mathrm{C}$ i.e. above the main chain transition to enable the swelling of bilayers.

The thermograms were recorded after three cycles (Tmin-Tmax $\left.5^{\circ} \mathrm{C} / \mathrm{min} ; \operatorname{Tmax}-\operatorname{Tmin} 5^{\circ} \mathrm{C} / \mathrm{min}\right)$. Each experiments was done triplicate.

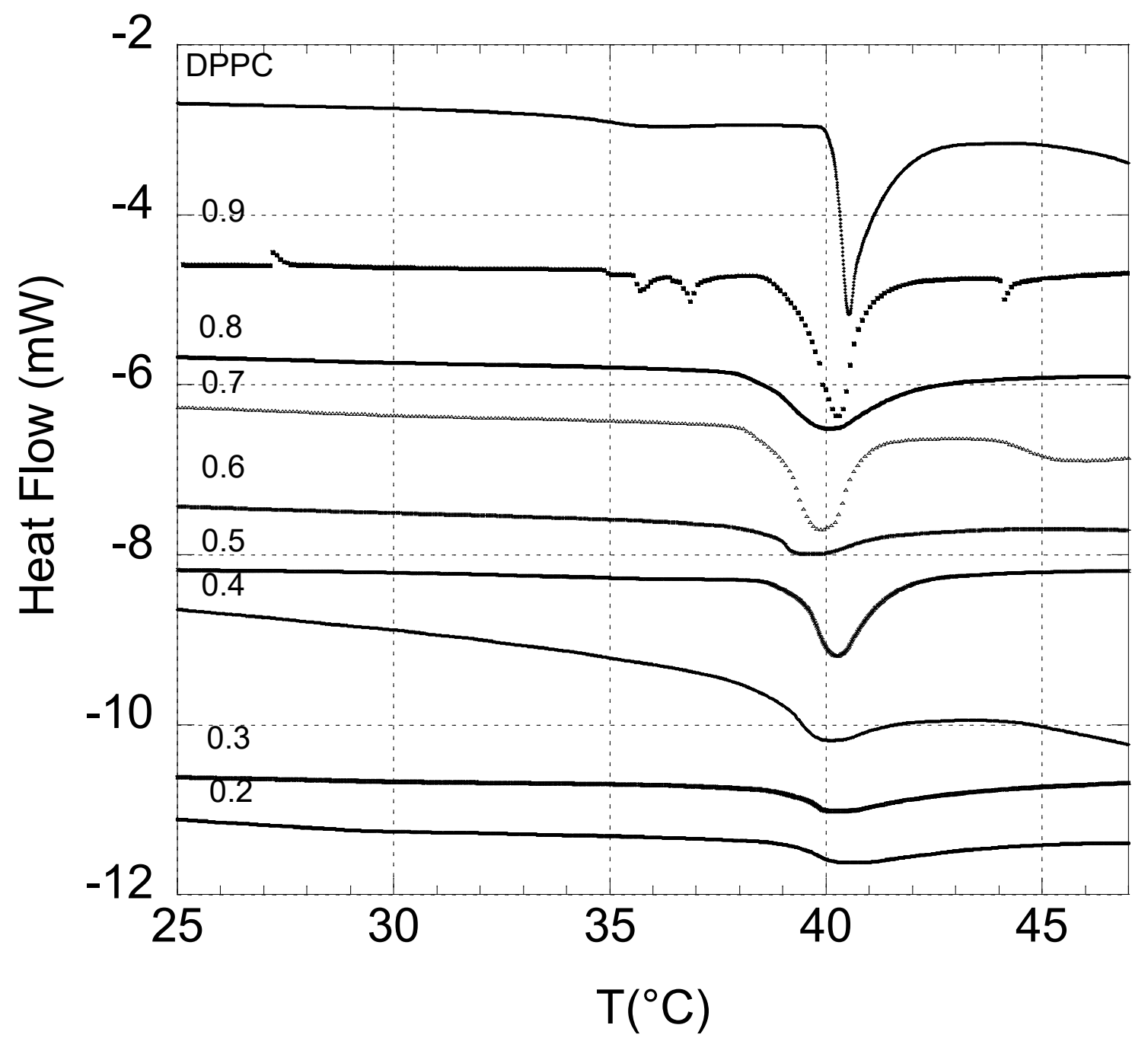

Figure S1: Thermograms obtained for vesicular suspensions of DPPC/PDMS-g-PEO 


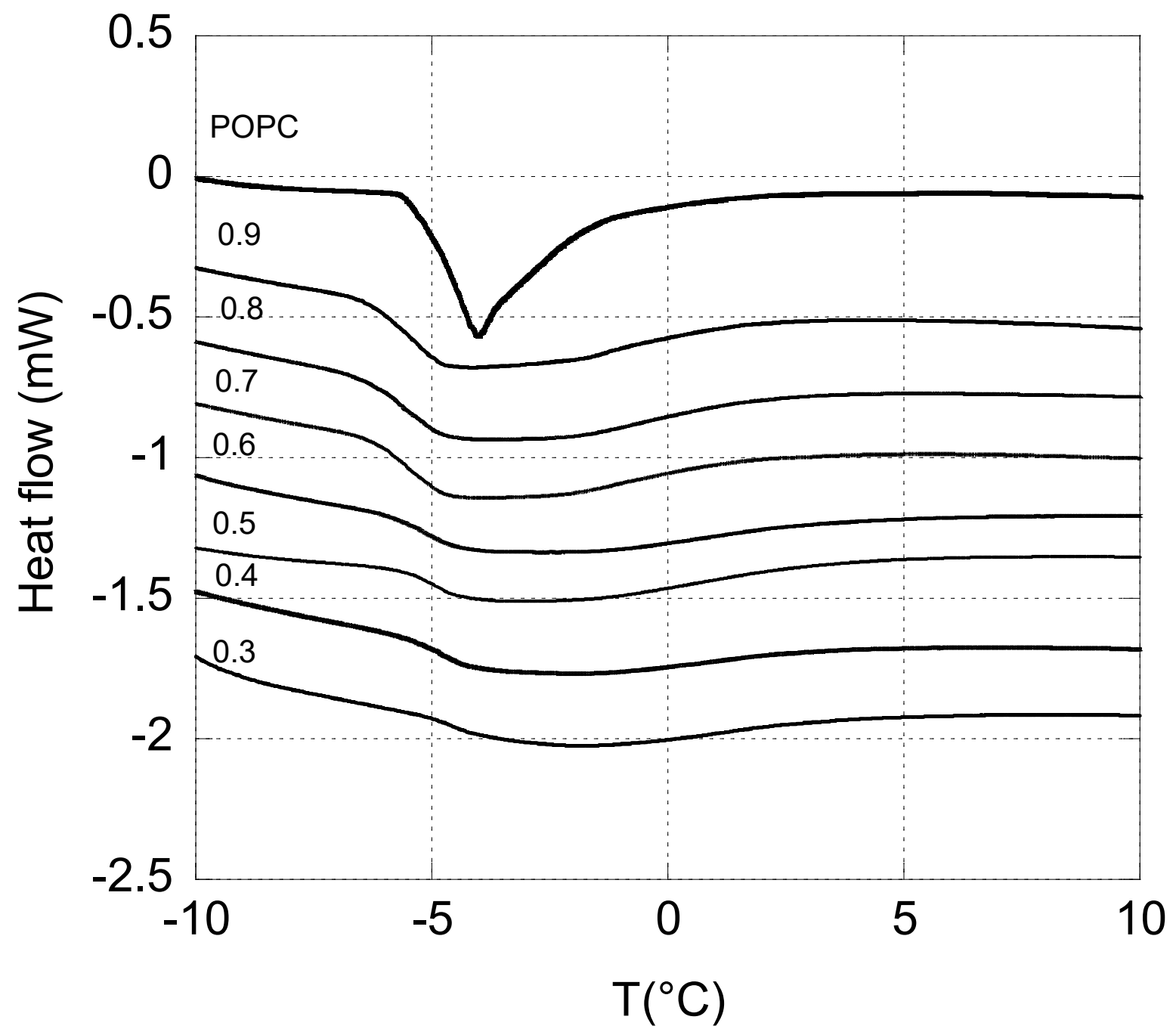

Figure S2: Thermograms obtained for vesicular suspensions of POPC/PDMS- $g$-PEO

\section{Tagging Copolymer with Fluorescein}

The PDMS-g-PEO in this study has been functionalized with Fluorescein using the hydroxyl end group of the PEO chains ( 2 hydroxyl end group in average for one PDMS- $g$-PEO copolymer chain)

The procedure was the following:

1-Mesylation of hydroxyl end group:

$1 \mathrm{~g}$ of PDMS-g-PEO (6.6e-4 mol of hydroxyl function) was dissolved in $20 \mathrm{ml}$ of dichloromethane and refrigerated at $0^{\circ} \mathrm{C}$. Under magnetic stirring, $185 \mu \mathrm{l}(1.33 \mathrm{e}-3 \mathrm{~mol})$ of triethylamine $(\mathrm{Et} 3 \mathrm{~N})$ was added at once therefore $56 \mu \mathrm{l}(7.3 \mathrm{e}-4 \mathrm{~mol})$ of mesyl chloride dissolved in $2 \mathrm{ml}$ of dichloromethane was added drop wise. The reaction proceeded overnight. Dichloromethane and mesylchloride in excess were removed under vacuum respectively a room temperature and at $60^{\circ} \mathrm{C}$. 


\section{2-Amination:}

$10 \mathrm{ml}$ of $28 \%$ aqueous ammonia was added directly to the flask containing PDMS- $g$-PEO mesylate. The lid was tightly closed and the reaction was vigorously stirred for 5 days at room temperature. The systems was purified by dialysis (membrane MWCO 25000Da) against MilliQ water, and dried under vacuum.

The final yield is $60 \%(w / w)$.

The amine content was checked by non aqueous titration using hydrobromic acid (1e-2M in acetic acid). The polymer was previously dissolved in a mixture of chloroform and acetic acid (50/50 vol.) and one drop of crystal violet was used as colorimetric indicator.

According to the titration, there is one amine group per PDMS-g-PEO copolymer chain

3-Coupling with N-hydroxysuccinimide (NHS)-Fluorescein

$95 \mathrm{mg}$ of PDMS- $\mathrm{g}$-PEO copolymer (3.16e-5mol of amine) was dissolved in $2 \mathrm{ml}$ of THF. $340 \mu \mathrm{l}$ of solution of diisopropylethylamine (DIPEA) at $12 \mathrm{mg} / \mathrm{ml}$ in THF $(3.16 \mathrm{e}-5 \mathrm{~mol})$ was added at once. $500 \mu \mathrm{l}$ of a solution of (NHS)-fluorescein at $40 \mathrm{mg} / \mathrm{ml}$ in DMSO $(4.22 \mathrm{e}-5 \mathrm{~mol})$ was added at once. The lid was tightly closed and the reaction was performed under gentle agitation during one day.

The purification was performed first by dialysis (membrane MWCO 25000Da) against basic water $\left(\mathrm{pH}^{\sim 10}\right)$ in order to facilitate the solubilisation of fluorescein in excess and therefore its removing. After 4 days, a slight yellow colour was still visible in dialysis bath. The polymer was then dried under vacuum, resuspended in basic water and purified using sephadex G25 column.

Finally the product was dried under vacuum and the final yield was evaluated at $65 \%(w / w)$.

The polymolecularity of the PDMS-g-PEO is not provided by the supplier (Dow corning) and its determination cannot be done by GPC using the most classical organic solvent THF. Indeed the refractive index increment of PDMS is very close to the one of THF and the resulting signal with RI detector is weak. This polymer cannot be detected with UV detector, and the molar mass is too weak to use light scattering detection.

Interestingly the resulting copolymer functionalized with fluorescein could be analysed by GPC in THF with UV detection as illustrated in the Figure S-3. The molar masses are extracted from a calibration with Polystyrene Standards

$\overline{M n}=3600 \mathrm{~g} / \mathrm{mol}, \overline{M w}=4760 \mathrm{~g} / \mathrm{mol} . \mathrm{lp}=1.32$ 

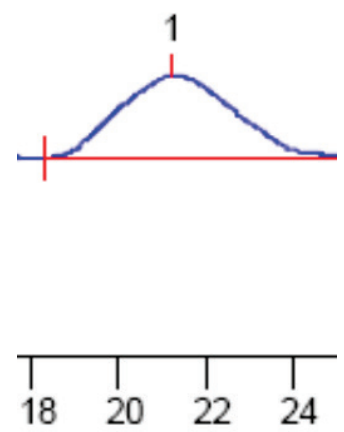

Elution volume (ml)

Figure S-3 Size exclusion Chromatogram PDMS-g-PEO-fluo acquired in THF

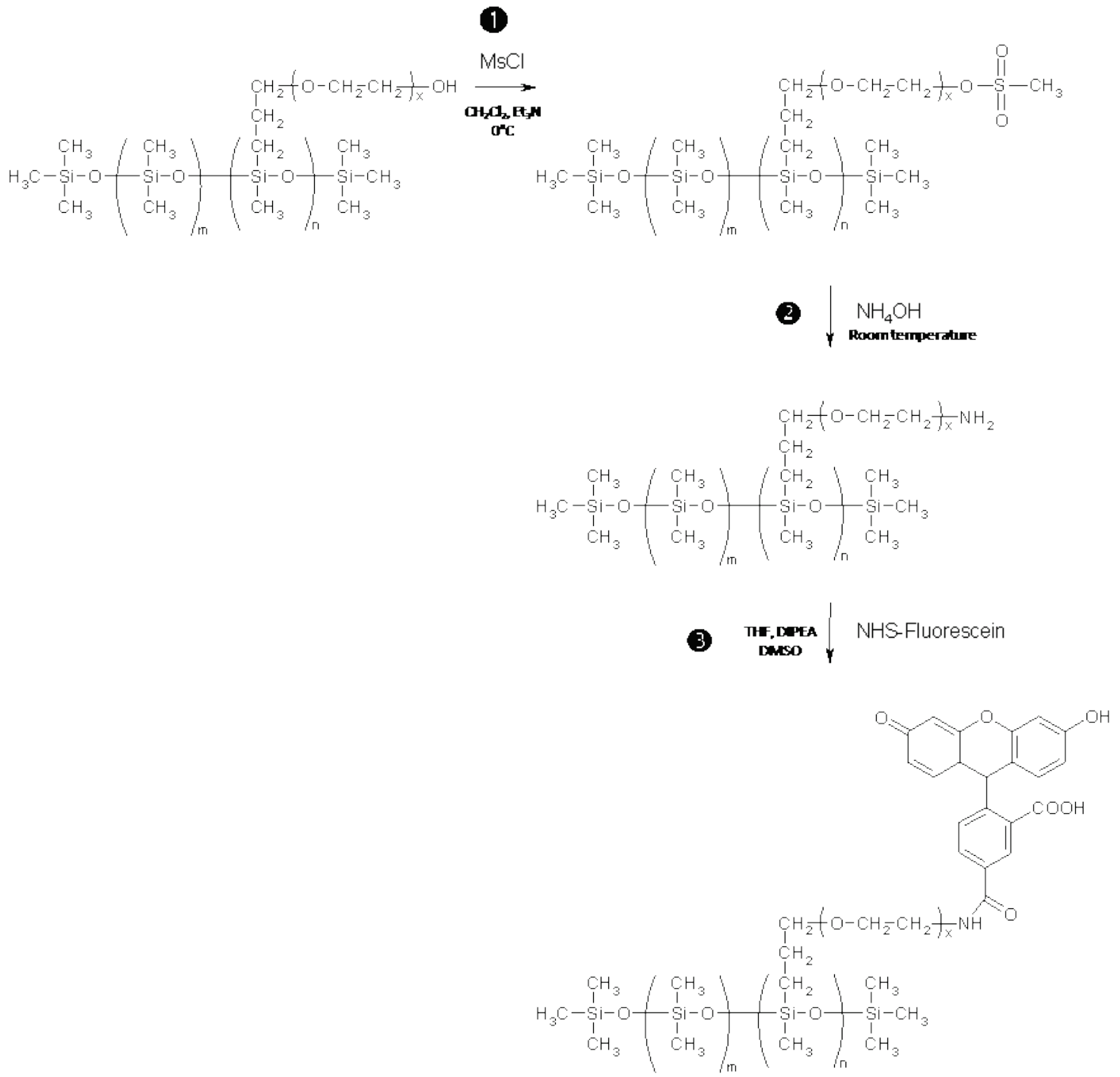

Scheme S-1: Reaction scheme of the tagging procedure of PDMS- $g$-PEO. 


\section{Evidence of the presence of lipid in polymer rich phases}
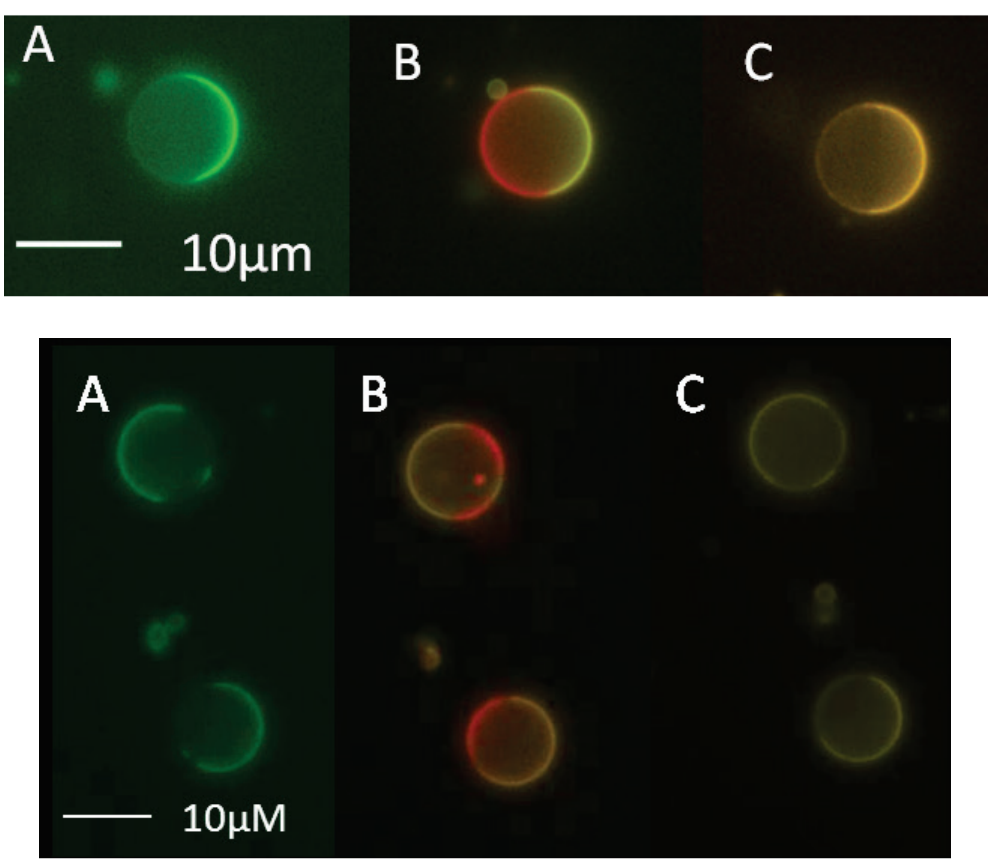

Figure S-4 Figure illustrating vesicles with heterogeneous distribution, with lipid present in the polymer-rich phase. Composition: 60 mol \% DPPC/40 mol \% PDMS-g-PEO.Filter sets: A, fluorescein;

$B$, triple-band; C, rhodamin.
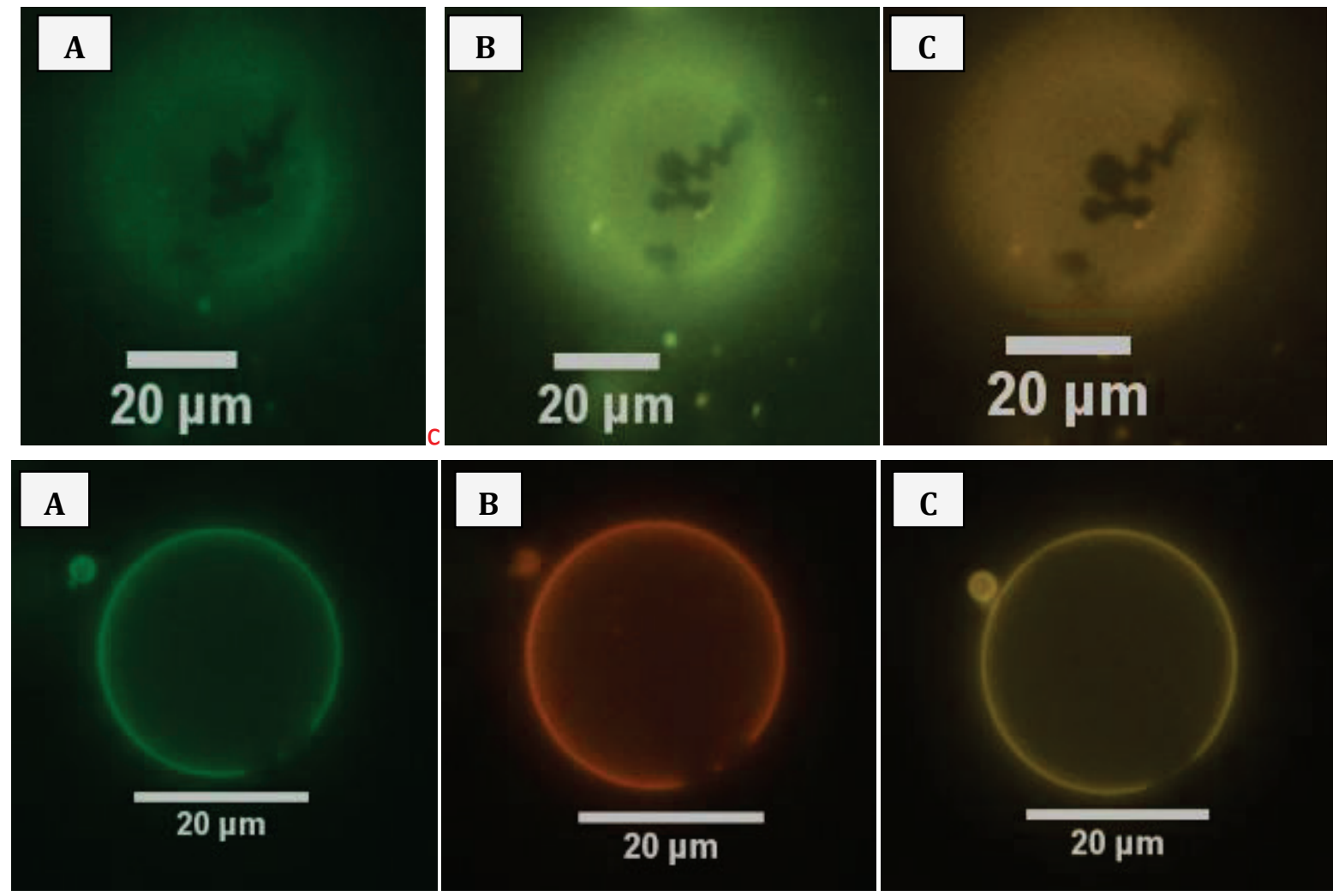

Figure S-5 Figure illustrating vesicles with heterogeneous distribution (here lipid domains in polymersome membrane), with lipid present in the polymer-rich phase. Composition: $25 \mathrm{~mol} \%$ DPPC/ 75mol\% PDMS-g-PEO. Filter sets: A, fluorescein; B, triple-band; C, rhodamin. 